\title{
Psychological Effects of the COVID-19 Pandemic: Perceived Stress, Anxiety, Work-family imbalance and Coping strategies among Healthcare Professionals in Khartoum State Hospitals, Sudan, 2021
}

Ibrahim Mahgoub

5th year Medical Student, Faculty of Medicine, University of Khartoum, Khartoum, Sudan

Amani Abdelrahman

Associate Professor of Pediatrics, Faculty of Medicine, Alzaiem Alazhari University, Khartoum, Sudan

Tibyan A Abdallah

University of Khartoum, Faculty of Medicine, Khartoum, Sudan

Khabab Abbasher Hussien Mohamed Ahmed

5th year Medical Student, University of Khartoum, Faculty of Medicine, Khartoum, Sudan

Mohammed Eltahier Abdalla Omer ( $\nabla$ Mohammedeltahier100@gmail.com )

MBSS, Gadarif University, Faculty of Medicine and Health Sciences, Gadarif, Sudan

https://orcid.org/0000-0002-7131-423X

\section{ElMuiz Abdelrahman}

5th year Medical Student, University of Khartoum, Faculty of Medicine, Khartoum, Sudan

\section{Zeinab Mohammed Amara Salih}

Assistant Professor of Community Medicine, Faculty of Medicine, University of Khartoum, Khartoum, Sudan

\section{Research Article}

Keywords: COVID-19, Stress, Anxiety, doctors and nurses, Sudan

Posted Date: June 2nd, 2021

DOl: https://doi.org/10.21203/rs.3.rs-583067/v1

License: (c) (1) This work is licensed under a Creative Commons Attribution 4.0 International License. Read Full License 
Version of Record: A version of this preprint was published at Brain and Behavior on August 1st, 2021. See the published version at https://doi.org/10.1002/brb3.2318. 


\section{Abstract}

Background: The 2019 novel coronavirus (COVID-19) is highly contagious and can spread a pandemic, so it is related to serious health issues and major public concerns, and is considered by the medical community to be the greatest concern because it is the greatest risk of infection .

Objective: To identify and assess the psychological effects of the COVID-19 Pandemic on healthcare professionals in Khartoum state hospitals 2021.

Materials and Methods: Generalized anxiety scale (GAD-7), Perceived Stress Scale (PSS-10) and Workfamily balance measure scale were used to assess the Psychological impact of Doctors and nurses working in four big hospitals in Sudan ,by an online questionnaire, analyzed by the statistical package for social science (SPSS) during February.

Results: Most of the participants had minimal to mild anxiety according to GAD-7 score, $121(35.2 \%)$ and $103(29.9 \%)$ respectively.

Using PSS-10 the cutoff point was determined as 19 as the mean for total score was 19.2 \pm 6.2 , accordingly more than half had high levels of stress (scored 19 and above) 189 (54.9\%). For the WorkFamily Balance scale, 10 was regarded as the cutoff point. There was a significant association between specialty and stress level $p$-value 0.032 . No significant correlations were found between age and stress level, neither between age and anxiety level $(r-.100$, $p$-value .064 and $r=-.022$, $p$-value .683 respectively).

Conclusion: More than half of healthcare professionals (54.9\%) showed high levels of stress. Most of the healthcare professionals had poor work-family balance $(60.2 \%)$.

\section{Introduction}

\section{Background}

In December 2019, a new strain of Coronavirus affected China and drastically spread around the world in an unprecedented manner, within a very short period. The World Health Organization renamed it Coronavirus disease 2019 (COVID-19), which is caused by severe acute respiratory syndrome coronavirus 2 (SARS-CoV-2) and declared a state of pandemic on 11 ${ }^{\text {th }}$ March 2020 (1). Since March 2020, the pandemic took hold in Sudan, as on the $13^{\text {th }}$ Sudan reported its first novel coronavirus case in Khartoum. A man who died on $12^{\text {th }}$ March after visiting the United Arab Emirates during the first week of March. Countries are racing to slow the spread of the virus by testing and treating patients, carrying out contact tracing, limiting to travel, quarantining citizens, and cancelling large gatherings such as sporting events, concerts, and schools. COVID-19 is transmitted chiefly by contact with infectious material (such as respiratory droplets) or with objects or surfaces contaminated by the causative virus, and is characterized especially by fever, may progress to cough, shortness of breath, pneumonia and respiratory failure. 
This produced a state of emergency in the country, and in Khartoum specifically. At present there are approximately thirty-four thousand eight hundred and eighty-nine $(34,889)$ people who got infected by the novel coronavirus in Sudan (2). Facing this unprecedented emergency, the government implemented extraordinary measures to control the viral transmission within the country as the exact mode of transmission was not yet confirmed by the World Health Organization (WHO) or the Federal Ministry of Health (FMOH). In this critical situation everyone's life changed due to restriction of movement and social contact . In particular, healthcare professionals uninterruptedly continued to work in this dangerous situation, acquiring the risk to be affected by COVID-19, therefore they might be considered as one of the most vulnerable categories of professionals to develop psychological stress and other mental health symptoms (3). A study of American adults found that one in four (25\%) were very worried about infection (4).

COVID-19 is an abbreviation that stands for (Coronavirus Disease of 2019) which was given by the World Health Organization. The International Committee on Taxonomy of Viruses (ICTV) suggested that this novel coronavirus was named SARS-CoV-2 due to the phylogenetic and taxonomic analysis of this novel coronavirus. According to the Johns Hopkins Coronavirus Resource Center (coronavirus.jhu.edu), as of January 2021, a total of approximately 98 million cases and 2 million deaths related to COVID-19 have been confirmed. This puts the spotlight on how severe getting infected with COVID-19 is, in addition to the lack of availability of effective therapy to date (5).

According to the Center for Disease Control and Prevention (CDC; https://www.cdc.gov/coronavirus/types.html), three out of the seven coronaviruses that epidemically outbroke throughout history in humans included SARS-CoV in 2002 (severe acute respiratory syndrome or SARS), and MERS-CoV in 2012 (Middle East respiratory syndrome or MERS), and currently SARS-CoV-2 (the current pandemic known as COVID-19) (6).Further research and studies of this novel coronavirus such as mechanism of infection and replication are required in order to develop effective therapeutic approaches and vaccination-mediated immunological memory, to eradicate the current and potential future pandemics (7). As future pandemics and more COVID waves may take place.

The possible transmission route of this novel coronavirus is person-to-person, which includes contact transmission by contacting the nasal, oral, and eye mucosal secretions of the infected individual, in addition to direct transmission by droplet inhalation when the infected individual produces nasal and/or oral aerosols during coughing or sneezing (8). Further research is still taking place to confirm the exact modes of transmission due to the uncertainty of many routes yet. Recent evidence has also suggested that COVID-19 can be transmitted to the fetus through placental circulation before birth in utero (9). It was also reported by several credible sources that droplets from sneezing or coughing can spread up to 6 feet (or 2 meters), emphasizing on the 6 feet social distancing criteria (10). The virus can deposit on many surfaces and can survive for days under favorable conditions depending on the particular surface it landed on. 
SARS-CoV-2 belongs to the Betacoronavirus genus and is a member of the Coron-Avirinae family (Genus: Betacoronavirus, Family: Coron-Avirinae).

The whole picture regarding clinical manifestations of SARS-CoV-2 is not yet clear, but the symptoms range from asymptomatic to mild to severe. Both the elderly and young patients can succumb to death depending on their underlying health conditions such as cardiovascular diseases, renal damage, liver diseases, diabetes, Parkinson's disease, and malignancies (11). Healthy individuals may recover from the viral infection within 2-4 weeks of treatment (12).

Currently there are several methods of diagnosing COVID-19, ranging from simple ones to more complex, with varying accuracy. The current and most used method for clinical diagnosis of SARS-CoV-2 (Corona Virus) is nucleic acid detection in nasopharyngeal and oropharyngeal swab samples by a reverse transcription-quantitative polymerase chain reaction (RT-qPCR) method (13).

Although RT-qPCR is highly specific, on some occasions it may also give false negative results because of sample cross-contamination or technical deficits. These issues cannot be ignored due to the inevitable consequences of a missed or wrong diagnosis, especially in the case of COVID-19 (14).

Favipiravir, Avigan or T-705, is a potential antiviral, as well as an anti-influenza drug approved in Japan for the treatment of influenza $A, B$, and $C$ viruses, including oseltamivir-resistant strains (15). Thirdly, Galidesivir, also known as BCX4430 or Immucillin-A, is a potential antiviral drug, and was originally developed to treat hepatitis $C$ (16).

Recently, mRNA-based vaccines BNT162 (developed by Pfizer/BioNTech) and mRNA-1273 (developed by Moderna Inc.) showed 95\% clinical efficacy in Phase 3 safety trials and this is considered a trusted percentage at which ministries of health can follow and approve. These two vaccine candidates are the front runners in the global vaccine race. Recently, BNT162 of Pfizer/BioNTech became the primary authority approved COVID-19 vaccine, to be followed by the mRNA-1273 of Moderna Inc. for vaccination within the us of America. The United Kingdom, Bahrain, Canada, and North American nation additionally approved the Pfizer/BioNTech COVID-19 vaccine's emergency use, despite the very fact that clinical trials don't seem to be fully conducted. China and Russia have already approved and are administering Corona Vac and artificial satellite $V$ vaccines, respectively, while not watching for the ultimate clinical trials results.According to data from the World Health Organization, approximately 162 candidate vaccines are currently undergoing preclinical evaluation, 52 of which are in clinical development. These strategies include inhibition of $S$ protein, proteases, mRNA, RNA-dependent-RNA-polymerase, whole virus vaccines, and antibody vaccines (17-20).

COVID-19 did not only impose adverse health and physical effects on the human body and the global healthcare system. As larger scale adverse effects showed up along the journey, it sparked fears of an impending economic crisis and recession. Social distancing, self-isolation and travel restrictions have lead to a remarkable decrease in demand for human resources across all economic sectors and caused many jobs to be lost within many infrastructures, at an unprecedented rate (21). 
The general situation in the world right now has triggered vast groups of people with or without predisposing factors to mental illness such as depression, anxiety, stress and lack of coping mechanisms. With healthcare professionals being the most vulnerable group to psychological effects and deficits, special attention is needed, and several studies have been conducted in this field over the past period. Excessive levels of stress represented a critical factor that could affect work environments and productivity (3). Depression or major depressive disorder is marked as one of the mood disorders, marked by episodes of depressed mood associated with loss of interest in daily activities (anhedonia) (22). It can be associated with sleep problems such as difficulty falling asleep, early morning awakenings (early and late insomnia), excessive sleepiness (hypersomnia), and an inability to experience pleasure (anhedonia). The factor that makes major depressive disorder alarming is that depression is associated with an increased mortality rate in patients with accompanying comorbidities such as diabetes, strokes and cardiovascular diseases (23), as well as increased risks of suicidal attempts.

Anxiety is defined as an individual emotional and physical fear response to perceived threat. Pathological anxiety occurs when symptoms are excessive, irrational, out of proportion to the trigger or without an identifiable trigger. The criteria for most anxiety disorders involve symptoms that cause clinically significant distress or impairment in social and/or occupational functioning (23).

Stress is defined as: "The bodily processes that result from circumstances that place physical or psychological demands on an individual" (24). It is known that stress takes place when an individual is unable to cope with a perceived past, present or future situation. Stress may trigger feelings of fear, incompetence, uselessness, anger, aggression and guilt, and if unresolved, may even lead to associated physical and psychological morbidities. However, owing to individual differences, the same situation that induces stress in one person may not have the same effect on another (25).

\section{Problem Statement}

Stress has a significant burden on healthcare professionals, which in turn reflects negatively on hospitals and the healthcare system in general, these two matters are regarded as inversely proportional. As excessive levels of stress represented a critical factor that could affect work environment and compromise performance, especially during an emergency (3). Furthermore, chronic work stress among healthcare professionals is associated with job satisfaction, physical health and post traumatic symptoms. As well as producing long term psychological consequences. Most importantly, this might cause major losses in human lives, hospital resources and long-term state strategies and goals.

The ongoing COVID-19 pandemic amplified stress and anxiety phenomena, and their implications among healthcare professionals remarkably. Kaiser Family Foundation (2020) found in a national survey that almost half (47\%) of US individuals who were sheltering in place reported higher rates of mental health problems due to worry and stress (4). Examples of observed and anticipated consequences of the pandemic included stress, feelings of helplessness, anxiety, depression, and loneliness. Moral injury has also been a topic of concern. This is a term used to define the psychological distress caused by a lack of 
or behavior that violates ethical or moral norms. Researchers warn of increasing rates of social problems such as domestic violence, suicide, alcohol, and substance abuse (4). The study aims to conduct a quantitative and qualitative measure of the consequences of the pandemic on healthcare professionals, in relation to the socio-demographic variables of everyone taking part in it.

This paper has laid sight and yielded good data that should aid in finding correlations between healthcare profession spectrums and stress levels, as well as providing recommendations that may remarkably decrease the consequences of the pandemic on healthcare professionals and the healthcare system.

\section{Justification (Rationale)}

Stress has put an intolerable burden on healthcare professionals because of the COVID-19 Pandemic, which directly affects the healthcare system in Sudan. This has caused and is still causing numerous adverse effects on the country. In terms of lack of concentration, medical errors by medical personnel including doctors, nurses and all healthcare professionals, less conservative use of medical resources, a negative working environment and eventually higher life deficits and losses.

Most of these adverse effects could be tackled through assessing the levels of stress, anxiety, workfamily imbalance and coping strategies among healthcare professionals and workers. Having assessed that, awareness could be raised on the matter and coping strategies can be amplified to aid this vulnerable population in getting over this pandemic's psychological effects and cope with it as much as possible. This study aims to assess the amount and types of stresses and anxieties that healthcare professionals experience, to identify different methods to tackle them and come out with feasible and logical recommendations, in regard to the country's situation and socioeconomic status. In addition to the fact that no similar study has been conducted or published in Sudan before.

\section{Objectives}

\section{General Objectives}

To identify and assess the psychological effects of the COVID-19 Pandemic on healthcare professionals in Khartoum state hospitals 2021.

\section{Specific Objectives}

To assess stress and anxiety levels amongst healthcare professionals during the COVID-19 pandemic

To determine the prevalence of work-family imbalance amongst healthcare professionals during the COVID-19 pandemic. 
To determine the coping strategies used by healthcare professionals to overcome work-related stress during the COVID-19 period.

To compare stress levels between doctors working in public hospitals and doctors working in private hospitals.

\section{Methodology \\ Study Design}

A descriptive cross-sectional hospital-based study

\section{Study Area (Setting)}

\section{1) Ibrahim Malik Teaching Hospital}

\section{Location:}

Alsahafa east, Khartoum, Khartoum, Sudan.

\section{Coverage Area:}

Covers Khartoum state mainly. It is a public hospital so everyone can get admitted and treated there as well.

Contains a COVID-19 Isolation ward.

\section{Staff:}

Regarding the working staff, there are 200 house officers, 50 medical officers, 10 Obs \& Gyne specialists, 4 pediatric specialists, 8 medicine specialists. 9 surgery specialists, 2 physiotherapists, 2 radiologists and 7 Obs \& Gyne consultants. 7 pediatric consultants, 9 medicine consultants, 7 surgery consultants. 226 Nurses and sisters work there.

\section{Units and Beds:}

Regarding the units there are 10 units: Radiology unit, Obstetrics and Gynecology unit, Pediatric unit, 8 Medicine units, 10 Surgery units, Accident and emergency unit, central care unit, Nursing unit, Physiotherapy unit and Central lab.

The number of beds in the hospital is 439 beds.

\section{2) Soba University Hospital}




\section{Location:}

Soba, Khartoum, Khartoum, Sudan.

\section{Background:}

The hospital was established in 1975 to provide health care to the residents of the region, as well as to train students of the Faculty of Medicine at the University of Khartoum and other universities, in addition to training doctors, house officers, medical officers, specialists, consultants, nursing personnel and laboratory sciences.

The hospital includes basic specialties in internal medicine, surgery, pediatrics, gynecology and obstetrics. The hospital also includes diagnostic and therapeutic units in laboratories, a blood bank, X-ray and ultrasound. It also includes intensive care units, operations complex, neonates, critical pregnancy, hemodialysis, mycetoma (which is the first center in the country for the treatment of mycelium), physiotherapy, plastic surgery, pediatric surgery, gastrointestinal and urology endoscopes. Soba Hospital is the first hospital in the Arab world to have a kidney transplant.

\section{Coverage Area:}

Covers Khartoum state. It is a public hospital so everyone can get admitted and treated there as well. Given its geographical location, it serves many patients from the outskirts of Khartoum especially Gezira State.

Contains a COVID-19 Isolation ward.

\section{3) Fedail Hospital}

\section{Location:}

Hawadith Street, Downtown, Khartoum, Sudan.

\section{Background:}

The experience of Fedail Hospital dates to 1992 when it was opened as Sudan Clinic, the first endoscopy oriented private clinic in Sudan, the first laparoscopic cholecystectomy in Sudan was performed in it.

Fedail Hospital is a newly established medical complex in Khartoum. In June 2003, the Fedail Medical Center was opened in the center of Khartoum on Hawadit Street and converted into Fedail Hospital with 120 beds with highly sophisticated medical equipment and infrastructure, more than 50 specialists from different specialties.

The hospital provides medical treatment for many international and local insurance companies through a special department in the hospital. 
Fedail Hospital is a modern hospital located in the heart of Khartoum, with 50 specialized clinics, inpatient facilities, accident and emergency unit, modern theatres, intensive care unit and advanced diagnostic and therapeutic facilities (28).

\section{Coverage:}

Fedail is a private hospital that serves all areas, mainly patients from Khartoum but anyone can get admitted and treated there as well. It is categorized as private but has insurance company privileges so patients with medical insurance are also treated there.

Contains a COVID-19 Isolation ward.

\section{Staff:}

250 Doctors and Nurses/sisters

\section{4) Al-Sha'ab Teaching Hospital}

\section{Location:}

Al-Mostashfa Street (Army Road), next to Khartoum Hospital and Stack Laboratories

\section{Background:}

Hospital was founded in 1959, opened by President Ibrahim Aboud.

Started as a chest hospital mainly that was specialized in the treatment and follow up of Tuberculosis patients. Later in the 1960s a cardiology unit was introduced to the hospital and served a large number of patients.

In the 1970 s a neurology unit was established along with a cardiac surgery unit.

Currently the hospital has a large number of beds, with emergency medicine departments, cardiology and chest emergency. House officers, medical officers, registrars, specialists and consultants practice there.

\section{Study Population}

Doctors and nurses working in Ibrahim Malik Hospital, Soba University Hospital, Al-Sha'ab Hospital, and Fedail hospital.

\section{Inclusion criteria:}

- Doctors and nurses working in Ibrahim Malik, Soba, Al-Sha'ab and Fedail hospitals.

- Nurses working in Ibrahim Malik, Soba, Al-Sha'ab and Fedail hospitals. 


\section{Exclusion criteria:}

- Doctors and nurses not working in the selected hospitals.

- Medical students and staff under training

- Nurses under training

- Individuals who refuse to fill the questionnaire.

\section{Sample Frame}

All doctors from different levels of practices including house officers, medical officers, registrars, specialists and consultants were included. With ranging specialties including medicine, surgery, pediatrics, obstetrics and gynecology, emergency medicine, anesthesia and intensive care. Nursing staff was included as well. The Healthcare professionals included were from Ibrahim Malik teaching hospital, Soba University Hospital, Al-Sha'ab Hospital and Fedail Hospital during the period of February 2021.

\section{Sample Size}

Equation used: (Cochran's formula)

$$
n=\frac{Z^{2} p q}{e^{2}}
$$

Where: $\mathrm{n}=$ minimum sample size required,

$\mathrm{z}=$ standard normal deviation $(1.95$ at $95 \%)$

$p=$ expected prevalence (estimated as 0.5 ), taken as $50 \%$ due to unknown population as previously conducted studies in this area

$q=1-p$

e=maximum acceptable random sampling error (here is $5 \%$ or 0.05 )

So my sample size is $=0.950625 /\left(0.05^{\wedge} 2\right)=\mathbf{3 8 0}$

Explanation: A total of 344 out of 380 samples was reached by the end of the data collection period, giving a response rate of $91 \%$. Could not reach the total sample size due to the strike of staff during the data collection period, limitation of time and refusal of staff to participate. 


\section{Sample Technique}

Simple random sampling technique was applied to this study. Simple random sampling was conducted on healthcare professionals (doctors from different levels and specialties) and nurses at Ibrahim Malik Hospital, Fedail Hospital, Soba Teaching Hospital and Al-Sha'ab Hospital during a period of three weeks. Data collection was carried out during February 2021.

\section{Data collection methods and tools}

\section{Data collection method:}

Data was collected using self-administrated paper form questionnaires, the sociodemographic information section and the coping strategies sections were author structured. While the remaining sections (GAD-7, PSS-10 and WFB) were standardized.

Paper form questionnaire was used. The questionnaire contained a total of 36 items/questions which took around 5 minutes to complete. The purpose of the study was explained to each participant and written consent was obtained at the beginning of the questionnaire, by individuals who agreed to participate. Participation was voluntary, a total of 344 healthcare professionals were included giving us a response rate of $91 \%$.

\section{Tools and measurement:}

1. Demographics: Participants were asked to fill out sociodemographic information including gender, age, occupation, level of practice, years of practice, specialty, working in COVID hospital or not, marital status and presence of children.

2. Generalized anxiety scale (GAD-7): a self-reported questionnaire for screening and severity measuring of generalized anxiety disorder (GAD) developed and published by Spitzer RL, Kroenke K, Williams JBW and Lowe B in 2006.

GAD-7 has seven items, which measure severity of various signs of GAD according to reported response categories with assigned points.

The GAD-7 items include: 1 ) nervousness; 2 ) inability to stop worrying; 3 ) excessive worry; 4) restlessness; 5) difficulty in relaxing; 6) easy irritation; and 7) fear of something awful happening. Assessment is indicated by the total score, which is made up by adding together the scores for the scale of all seven items. Each item has four responses which were scored as 0, 1, 2 and 3. (29)

3. Perceived Stress Scale (PSS-10): a self-reported questionnaire developed to measure the degree to which situations in one's life are appraised as stressful. The PSS was published in 1983 and has 
become one of the most widely used psychological instruments for measuring nonspecific perceived stress. It has been used in studies assessing the stressfulness of situations, the effectiveness of stress-reducing interventions, and the extent to which there are associations between psychological stress and psychiatric and physical disorders.

PSS-10 has 10 items, and each item has five responses which were scored as 0, 1, 2, 3, 4 (30).

4. Work-family balance measure scale: a self-reported 3-item questionnaire developed and published by Matthews, R. A., Kath, L. M., and Barnes-Farrell, J. L. in 2010 to provide a predictive measure of workfamily conflict, that may occur as a result of external factors such as occupation. This scale has 3 items, and each item has five responses which were scored as 1, 2, 3, 4, 5.

5. Section to determine the coping strategies used by healthcare professionals to overcome stresses. (An author-structured questionnaire).

(For content validity, a pilot study was conducted. The questionnaire was pretested on 10 volunteer healthcare professionals. They commented on the relevance and ambiguity of items)

\section{Study Variables}

\section{Independent Variables:}

- Gender

- Age

- Healthcare profession and level of practice (house officer, consultant, etc.)

- Specialty

- Years of practice

- Working in COVID hospital or not

- Marital status

- Presence of children

\section{Dependent Variables:}

- Stress level score

- Anxiety level score

- Work-family balance score

- Coping strategy used

\section{Data management and Analysis}


The data collected were entered into Microsoft office excel database and analyzed using Statistical Package for Social Science (SPSS v.26) system.

Results were displayed in the form of tables, charts, graphs and text where relevant.

\section{Duration of Study}

One month (Data was collected during February 2021)

\section{Ethical Considerations}

Ethical approval was obtained from the department of community medicine, University of Khartoum.

Permission was obtained from the State Ministry of Health to collect data from Ibrahim Malik Teaching Hospital and Al-Sha'ab Hospital.

Permission was obtained from the Management of private medical institutions to collect data in Fedail hospital.

Permission was obtained from all the selected hospital administrators to collect data within the premises.

Written and verbal consent was obtained from the recipients at the beginning of the questionnaire.

No information that leads to the identification of any individual was taken as the confidentiality and anonymity of the participants were the highest priority.

\section{Results}

In this study a total of 344 Healthcare workers were included. Two thirds of them were female 230 $(66.9 \%)$ and the majority $241(70.1 \%)$ were from the age group $20-30$ years. More than half of the participants $183(53.2 \%)$ were doctors from different levels of practice and specialties and the remaining were nurses. The vast majority mentioned that they are working in isolation centers 323 (93.9\%). 243 (70.6\%) of them were single and 252 (73.2\%) did not have children.

Most of the participants had minimal to mild anxiety according to GAD-7 score, 121 (35.2\%) and 103 (29.9\%) respectively.

Using PSS-10 the cutoff point was determined as 19 as the mean for total score was 19.2 \pm 6.2 , accordingly more than half had high levels of stress (scored 19 and above) 189 (54.9\%). For the WorkFamily Balance scale, 10 was regarded as the cutoff point and accordingly those who scored less than 10 were regarded as having good work-family balance and accordingly most of them had poor balance $207(60.2 \%)$. 
In terms of coping strategies, the most commonly mentioned coping strategies used by the participants when undergoing stressful conditions were Turning to religion (sample item: "I pray more than usual") and Positive attitude (sample item: "I look for something good in what is happening), 237 (68.9\%) and $217(63.1 \%)$ respectively.

A highly significant correlation was found between stress level and years of practice, that is those with more experience tend to have lower level of stress $r=-.160$, $p$-value .003 . Also, a significant negative correlation was found between years of experience and anxiety level,

$r=-.137, p$-value .011

No significant correlations were found between age and stress level, neither between age and anxiety level $(r-.100$, p-value .064 and $r=-.022$, p-value .683 respectively).

There was a significant association between specialty and stress level p-value 0.032 , with those working in the Emergency Medicine departments being the commonest group with low stress level. There was no significant association between anxiety level and specialty p-value 0.134 .

Difference was tested based on hospital type whether private or public and accordingly no significant difference was found whether on stress level or anxiety level p-value 0.893 and 0.802 , respectively.

Also, no significant difference was found in stress level and anxiety level according to type of hospital whether isolation center or not, $\mathrm{p}$-value 0.818 and 0.437 , respectively.

Chi-squire and likelihood ratio were used for association, Spearman for correlation and Mann-Whitney $\mathrm{U}$ test for difference.

In all tests, p-value was regarded significant when below or equal to 0.05 .

Table 3.1: Sociodemographic information of the healthcare professionals who participated in the study to assess the psychological effects of the COVID-19 pandemic on healthcare professionals. 


\begin{tabular}{|c|c|c|c|}
\hline & & $\mathrm{n}$ & $\%$ \\
\hline \multirow[t]{2}{*}{ Gender } & Male & 114 & 33.1 \\
\hline & Female & 230 & 66.9 \\
\hline \multirow[t]{4}{*}{ Age (years) } & $20-30$ & 241 & 70.1 \\
\hline & $31-40$ & 71 & 20.6 \\
\hline & $41-50$ & 21 & 6.1 \\
\hline & Above 50 & 11 & 3.2 \\
\hline \multirow[t]{6}{*}{ Position } & House officer & 56 & 16.3 \\
\hline & Medical officer & 52 & 15.1 \\
\hline & Registrar & 49 & 14.2 \\
\hline & Specialist & 17 & 4.9 \\
\hline & Consultant & 9 & 2.6 \\
\hline & Nurse & 161 & 46.8 \\
\hline \multirow[t]{5}{*}{ Years of practice } & $1-5$ & 229 & 66.6 \\
\hline & $6-10$ & 71 & 20.6 \\
\hline & $11-15$ & 12 & 3.5 \\
\hline & $16-20$ & 15 & 4.4 \\
\hline & More than 20 & 17 & 4.9 \\
\hline \multirow[t]{2}{*}{ Do you work in a hospital with a COVID-19 isolation center? } & Yes & 323 & 93.9 \\
\hline & No & 21 & 6.1 \\
\hline \multirow[t]{3}{*}{ Marital status } & Single & 243 & 70.6 \\
\hline & Married & 93 & 27.0 \\
\hline & Divorced & 8 & 2.3 \\
\hline \multirow[t]{2}{*}{ Do you have children? } & Yes & 91 & 26.8 \\
\hline & No & 252 & 73.2 \\
\hline
\end{tabular}

Table 3.2: Prevalence of anxiety amongst healthcare professionals who participated in the study to assess the psychological effects of the COVID-19 pandemic on healthcare professionals using GAD-7 


\begin{tabular}{|lll|}
\hline & $\mathrm{n}$ & $\%$ \\
\hline Minimal & 121 & 35.2 \\
\hline Mild & 103 & 29.9 \\
\hline Moderate & 78 & 22.7 \\
\hline Severe & 42 & 12.2 \\
\hline
\end{tabular}

Table 3.3: Prevalence of stress amongst healthcare professionals who participated in the study to assess the psychological effects of the COVID-19 pandemic on healthcare professionals using PSS-10

\begin{tabular}{|lll|}
\hline & $\mathrm{n}$ & $\%$ \\
\hline Low stress & 155 & 45.1 \\
\hline High stress & 189 & 54.9 \\
\hline
\end{tabular}

Table 3.4: Levels of Work-family balance among healthcare professionals who participated in the study to assess the psychological effects of the COVID-19 pandemic on healthcare professionals using a 3item questionnaire

\begin{tabular}{|lll|}
\hline & $\mathrm{n}$ & $\%$ \\
\hline Good balance & 137 & 39.8 \\
\hline Poor balance & 207 & 60.2 \\
\hline
\end{tabular}

Table 3.5: Coping strategies used by healthcare professionals who participated in the study to assess the psychological effects of the COVID-19 pandemic on healthcare professionals during the stressful conditions of the COVID-19 pandemic

\begin{tabular}{|lll|}
\hline & $\mathrm{n}$ & $\%$ \\
\hline I seek Social Support (Get advice from someone) & 196 & 57.0 \\
\hline I refuse to believe all that has happened (Avoidance/Denial Strategy) & 36 & 10.5 \\
\hline I look for something good in the situation (Positive attitude) & 217 & 63.1 \\
\hline I try to deal with the situation and solve the problem & 186 & 54.1 \\
\hline I pray more than often (Turning to religion) & 237 & 68.9 \\
\hline I listen to music & 117 & 34.0 \\
\hline I perform exercises & 75 & 21.8 \\
\hline
\end{tabular}




\section{Discussion}

This study aimed to analyze the psychological effects of COVID-19 on healthcare professionals, that is including: perceived stress, anxiety, work-family balance and coping strategies. Analyzing the relationship between risk factors, protective factors and distress levels. The overall response rate reached was $91 \%$, where a total of 344 Healthcare professionals were included. Specifically, the role of some sociodemographic variables was investigated (age, gender, occupation, level of practice, specialty, years of practice, working in COVID-19 hospital or not, marital status, having or not children) (Table 3.1) to determine their effect on stress and anxiety levels, along with the coping strategies used to overcome these stresses. Due to the cross-sectional nature of this study, no causal conclusions can be drawn or confirmed. Despite this point, the findings may lead to understanding of stress and anxiety levels, workfamily balance levels and coping strategies implemented by healthcare professionals during the COVID19 outbreak.

In general, most of the participants had minimal to mild anxiety according to GAD-7 score, 121 (35.2\%) and $103(29.9 \%)$ respectively. (Table 4.2 ) In addition, more than half of the participants had high levels of stress (scored 19 and above) 189 (54.9\%). (Table 3.3)

Regarding the sociodemographic variables, a highly significant correlation was found between stress level and years of practice, that is those with more experience tend to have lower levels of stress $r=$ -0.160, p-value 0.003. In addition, a significant negative correlation was found between years of experience and anxiety level, $r=-0.137, p$-value 0.011 . This result was in line with previous literature on the theme (Elbay et al. 2020) in Turkey and another literature (Amin et al. 2020) in Pakistan. The results of the studies revealed that having less professional experience was associated with higher scores of stress and anxiety (27). Probably explained by the fact that the more a healthcare professional has been practicing in the medical field, the more exposed they have become to different stressors which leads to gradual desensitization, therefore future triggers such as the current pandemic would be less of a stimulant to high stress and anxiety levels. There was no significant difference found in levels of stress and anxiety between the less experienced and more experienced staff in a study conducted in Italy (3).

Results showed no significant correlation between age and stress level, and age and anxiety level ( $r$ -0.100 , $p$-value 0.064 and $r=-0.022$, $p$-value 0.683 respectively). This result is not consistent with a recent research (Amin et al. 2020) that found that the prevalence of anxiety and stress was significantly higher among younger physicians (27). It is argued that lack of experience forms a considerable part of this theory.

There was a significant association between specialty and stress level p-value 0.032 , with those working in the ER being the commonest group with low stress levels. As it is commonly argued that medical staff working in fields with constant high stress levels are better adapted to perform adequately regardless of the unexpected situations taking place, in this study case COVID-19 is being the stressful trigger that staff in specialties other than Emergency medicine are not well accustomed to. This study result was not in line with two studies conducted in China and Pakistan, where it was found that emergency medicine 
healthcare professionals and frontline health workers engaged in direct diagnosis, treatment and care of patients with COVID-19 were at a higher risk of showing symptoms of stress, anxiety and depression. Where being in contact with more than five COVID-19 confirmed cases per day was associated with anxiety and depression both in univariable and multivariable analysis $(26,27)$. This difference in results between Sudan and other countries like China and Pakistan is arguably due to the fact that the healthcare system in Sudan is already weak which exposes medical personnel to working in very limited infrastructures under high levels of pressure, nevertheless, they still manage to perform their tasks at adequate levels. Where in other countries with better infrastructures and support systems, events like the pandemic may form huge changes in working environments which can be hard to adapt to quickly. This study also found that there was no significant association between anxiety level and specialty, p-value 0.134. Again, due to the cross-sectional nature of this study no causal conclusions in terms of relationships between specialty and anxiety levels could be drawn.

A further objective of the study was to explore if working in a private hospital compared to a governmental hospital would affect levels of psychological distress. Difference was tested and surprisingly no significant difference was found whether on stress level or anxiety level, $p$-value 0.893 and 0.802 , respectively. Which was not in line with this study's hypothesis which stated that healthcare professionals working in private hospitals would show lower levels of stress and anxiety, due to the known fact that in Sudan the private healthcare sector is better equipped with personal protective equipment, has better medical infrastructure and provides better administrative and psychological support to healthcare professionals in general. But this result is self-explanatory as most healthcare professionals in Sudan work in a rotating manner between public sectors for training, and private sectors for financial income. Which makes the participants' responses prone to bias or facilitates for lack of accuracy and recall bias. The results are not consistent with other results and this may be due to the different working environments and infrastructures in which these researches were conducted.

In this study the most common coping strategies mentioned by participants when undergoing stressful situations such as the COVID-19 pandemic were "Praying more than usual (turning to religion)" and looking for something good in the situation (positive attitude), 237 (68.9\%) and 217 (63.1\%) respectively. This finding was consistent with previous studies in Italy (Babore at al. 2020) and in Saudia Arabia (Khalid et al. 2016) that found that a positive attitude in the workplace is the strategy with the biggest impact in reducing stress and anxiety levels $(3,31)$. The finding was also consistent with a study conducted in Oman during the COVID-19 outbreak (Badahdah et al. 2020), which found that the most used coping mechanism was "Turning to religion" (4). This is explained by the fact that countries like Sudan, Oman and middle eastern countries in general are highly influenced and guided by religion, which is widely used as a form of meditation, relaxing and gaining spiritual support.

\section{Conclusion And Recommendations}

\section{Conclusion:}


- Study found that most of the participants had minimal to mild anxiety, (35.2\%) and (29.9\%) respectively.

- More than half of healthcare professionals (54.9\%) showed high levels of stress.

- Most of the healthcare professionals had poor work-family balance (60.2\%).

- The most common coping strategies used by healthcare professionals during the COVID-19 pandemic were "Praying more than usual (turning to religion)" and looking for something good in the situation (positive attitude).

- There was no significant difference in stress levels between healthcare professionals working in public and private hospitals.

\section{Recommendations:}

- To provide administrative and psychological support to healthcare professionals

- To provide frequent and accurate updates on the COVID-19 situation to healthcare professionals

- To conduct routine screening of healthcare professionals for stress and anxiety

- To support and encourage a positive attitude in the work environment and turning to religion, as coping strategies during stressful times.

- To address the mental health of healthcare professionals working during the COVID-19 pandemic and future wise.

\section{Limitations:}

- Due to the cross-sectional nature of the study, the causal relationship between stress levels and sociodemographic variables could not be completely drawn or confirmed.

- Due to the cross-sectional nature of the study participants may be misclassified due to changes in stress exposure or poor memory of earlier exposure.

- Data not $100 \%$ reliable as self-reported information/questionnaires are prone to recall bias.

- At the time of the study, doctors were on strike therefore there was a compromised representation of the different levels of practice in the sample.

\section{Declarations}

\section{Availability of data and materials}

The materials datasets used and/or analyzed during this study are available from the corresponding author on reasonable request.

\section{Competing interests}


The authors declare that they have no competing interests.

\section{Funding}

This research did not receive any specific grant from funding agencies in the public, commercial, or notfor-profit sectors.

\section{Authors' contributions}

All authors participated in planning the study, data collection, results and discussion sections.

\section{References}

1. World Health Organization. COVID-19 Weekly Epidemiological Update: Data as received by WHO from national authorities, as of 29 November 2020, 10 am CET. 2020;(November). Available from: https://www.who.int/publications/m/item/weekly-epidemiological-update-1-december-2020

2. Figures K. Weekly Operational Update on COVID-19. World Heal Organ. 2021;(January):1-11.

3. Babore A, Lombardi L, Viceconti ML, Pignataro S, Marino V, Crudele M, et al. Psychological effects of the COVID-2019 pandemic: Perceived stress and coping strategies among healthcare professionals. Psychiatry Res [Internet]. 2020;293(August):113366. Available from: https://doi.org/10.1016/j.psychres.2020.113366

4. Badahdah A, Khamis F, Al Mahyijari N, Al Balushi M, Al Hatmi H, Al Salmi I, et al. The mental health of health care workers in Oman during the COVID-19 pandemic. Int J Soc Psychiatry. 2020;

5. Statement $C$. The species Severe acute respiratory syndromerelated coronavirus: classifying 2019nCoV and naming it SARS-CoV-2. Nat Microbiol. 2020;5(March).

6. Wiersinga WJ, Rhodes A, Cheng AC, Peacock SJ, Prescott HC. Pathophysiology, Transmission, Diagnosis, and Treatment of Coronavirus Disease 2019 (COVID-19) A Review. 2020;2019:1-13.

7. Chilamakuri R, Agarwal S. COVID-19: Characteristics and Therapeutics. Cells. 2021;10(2):1-29.

8. Chan JF, Yuan S, Kok K, To KK, Chu H, Yang J, et al. A familial cluster of pneumonia associated with the 2019 novel coronavirus indicating person-to-person transmission: a study of a family cluster. Lancet [Internet]. 2020;395(10223):514-23. Available from: http://dx.doi.org/10.1016/S01406736(20)30154-9

9. Rimmer A. Covid-19: doctors in final trimester of pregnancy. 2020;1173(March):2118. Available from: http://dx.doi.org/doi:10.1136/bmj.m1173

10. Setti L, Passarini F, Gennaro G De, Barbieri P, Piscitelli P, Miani A. Airborne Transmission Route of COVID-19: Why 2 Meters / 6 Feet of Inter-Personal Distance Could Not Be Enough. 2020;

11. Harcourt J, Tamin A, Lu X, Kamili S, Sakthivel SK, Murray J, et al. Severe acute respiratory syndrome coronavirus 2 from patient with coronavirus disease, United States. Emerg Infect Dis. 2020;26(6):1266-73. 
12. Huang C, Wang Y, Li X, Ren L, Zhao J, Hu Y, et al. Clinical features of patients infected with 2019 novel coronavirus in Wuhan, China. Lancet. 2020;395(10223):497-506.

13. Udugama B, Kadhiresan P, Kozlowski HN, Malekjahani A, Osborne M, Li VYC, et al. Diagnosing COVID-19: The Disease and Tools for Detection. ACS Nano. 2020;14(4):3822-35.

14. Li X, Geng M, Peng Y, Meng L, Lu S. Molecular immune pathogenesis and diagnosis of COVID-19. J Pharm Anal [Internet]. 2020;10(2):102-8. Available from: https://doi.org/10.1016/j.jpha.2020.03.001

15. Agrawal U, Raju R, Udwadia ZF. Favipiravir: A new and emerging antiviral option in COVID-19. Med J Armed Forces India [Internet]. 2020;76(4):370-6. Available from: https://doi.org/10.1016/j.mjafi.2020.08.004

16. Evans GB, Tyler PC, Schramm VL. Immucillins in Infectious Diseases. ACS Infect Dis. 2018;4(2):10717.

17. Bennet BM, Wolf J, Laureano R, Sellers RS. Review of Current Vaccine Development Strategies to Prevent Coronavirus Disease 2019 ( COVID-19). 2020;

18. Dutta AK. Vaccine Against Covid-19 Disease - Present Status of Development. 2020;

19. Ita K. Coronavirus Disease ( COVID-19): Current Status and Prospects for Drug and Vaccine Development. Arch Med Res [Internet]. 2020;1-10. Available from: https://doi.org/10.1016/j.arcmed.2020.09.010

20. Kaur SP, Gupta V. COVID-19 Vaccine: A comprehensive status report. Virus Res [Internet]. 2020;288(July):198114. Available from: https://doi.org/10.1016/j.virusres.2020.198114

21. Nicola M, Alsafi Z, Sohrabi C, Kerwan A, Al-Jabir A, losifidis C, et al. The socio-economic implications of the coronavirus pandemic (COVID-19): A review. Int J Surg [Internet]. 2020;78(April):185-93. Available from: https://doi.org/10.1016/j.jjsu.2020.04.018

22. Galea S, Merchant RM, Lurie N. The Mental Health Consequences of COVID-19 and Physical Distancing [Internet]. 2020 [cited 2021 Mar 15]. Available from: https://jamanetwork.com/journals/jamainternalmedicine/fullarticle/2764404

23. Ganti L, Kaufman MS, Blitzstein SM. FIRST AID PSYCHIATRY.

24. Selye H. Stress theory [Internet]. 1973 [cited 2021 Jan 10]. Available from: https://en.wikipedia.org/wiki/Hans_Selye

25. Naidoo SS, Wyk J Van, Higgins-opitz SB, Moodley K. An evaluation of stress in medical students at a South African university An evaluation of stress in medical students at a South African university. South African Fam Pract [Internet]. 2014;6190:1-5. Available from: http://dx.doi.org/10.1080/20786190.2014.980157

26. Liu CY, Yang YZ, Zhang XM, Xu X, Dou QL, Zhang WW. The prevalence and influencing factors for anxiety in medical workers fighting COVID-19 in China: A cross-sectional survey. medRxiv. 2020;(May 2020).

27. Amin F, Sharif S, Saeed R, Durrani N, Jilani D. COVID-19 pandemic- knowledge, perception, anxiety and depression among frontline doctors of Pakistan. BMC Psychiatry. 2020;20(1):1-9. 
28. Fedail Hospital. Fedail Hospital About Us [Internet]. 2017. [cited 2021 Mar 25]. Available from: http://fedailhospital.com/about-us/

29. Spitzer RL, Kroenke K WJ and LB. Generalized Anxiety Disorder-7 Scale. 2006; Available from: https://en.wikipedia.org/wiki/Generalized_Anxiety_Disorder_7

30. Cohen, Hewitt. Perceived Stress Scale-10. 1983; Available from: https://en.wikipedia.org/wiki/Perceived_Stress_Scale

31. Imran K. Healthcare Workers Emotions, Perceived Stressors and Coping Strategies During a MERSCoV Outbreak. 2016;14(1):7-14.

\section{Figures}

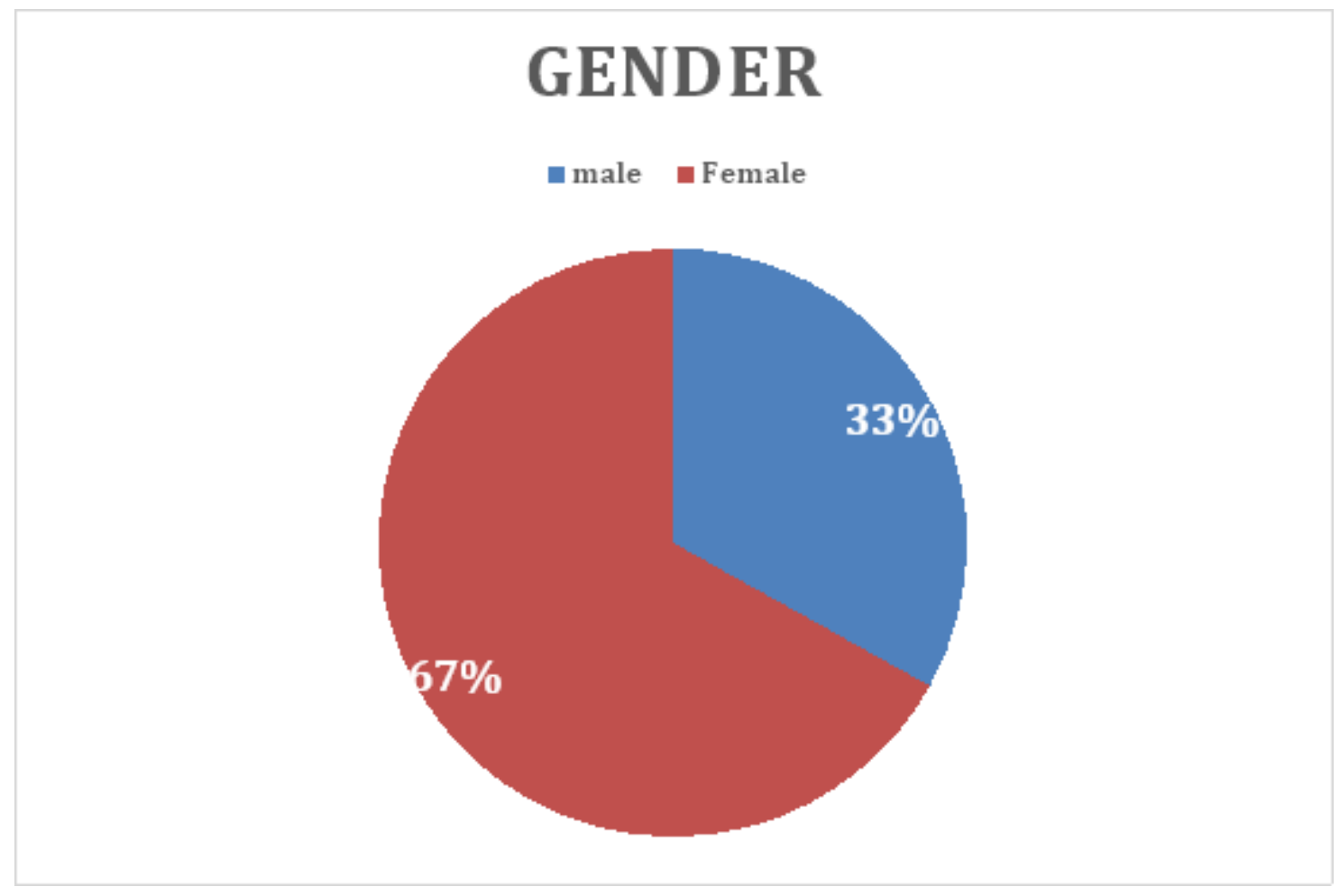

\section{Figure 1}

Figure 3.1: This figure shows the percentage of males and females who participated in the study to assess the psychological effects of the COVID-19 pandemic on healthcare professionals, Khartoum state hospitals, February $2021(\mathrm{~N}=344)$ 


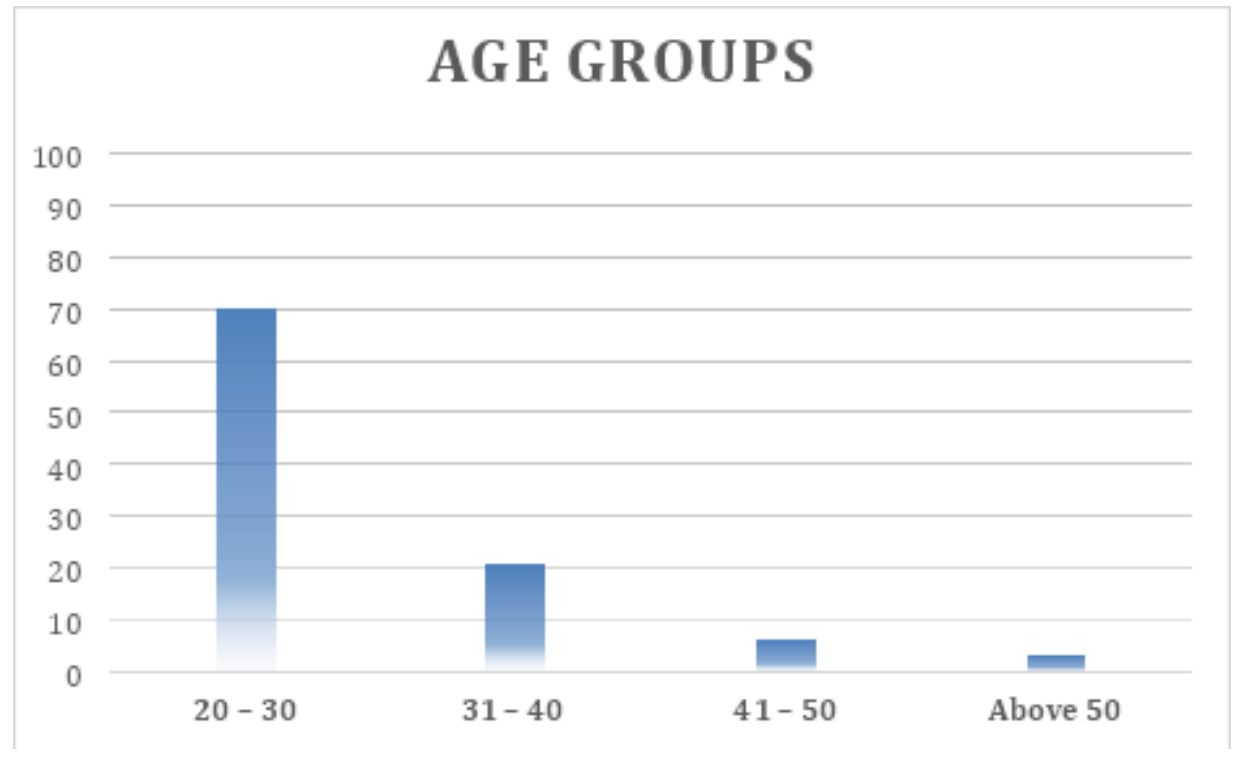

\section{Figure 2}

Figure 3.2: This figure shows the distribution of healthcare professionals according to age group (percentages) who participated in the study to assess the psychological effects of the COVID-19 pandemic on healthcare professionals, Khartoum state hospitals, February $2021(\mathrm{~N}=344)$

\section{Figure 3}

Figure 3.3: This figure shows the distribution of healthcare professionals according to occupation/level of practice (percentages) who participated in the study to assess the psychological effects of the COVID19 pandemic on healthcare professionals, Khartoum state hospitals, February $2021(\mathrm{~N}=344)$

\section{Figure 4}

Figure 3.4: This figure shows the distribution of healthcare professionals according to years of practice (percentages) who participated in the study to assess the psychological effects of the COVID-19 pandemic on healthcare professionals, Khartoum state hospitals, February $2021(\mathrm{~N}=344)$

\section{Figure 5}

Figure 3.5: Figure showing the percentage of healthcare professionals working in hospitals with COVID19 isolation centers who participated in the study to assess the psychological effects of the COVID-19 pandemic on healthcare professionals, Khartoum state hospitals, February 2021 ( $N=344$ )

\section{Figure 6}


Figure 3.6: This figure shows the distribution of healthcare professionals according to marital status (percentages) who participated in the study to assess the psychological effects of the COVID-19 pandemic on healthcare professionals, Khartoum state hospitals, February $2021(\mathrm{~N}=344)$

\section{DO YOU HAVE CHILDREN?}

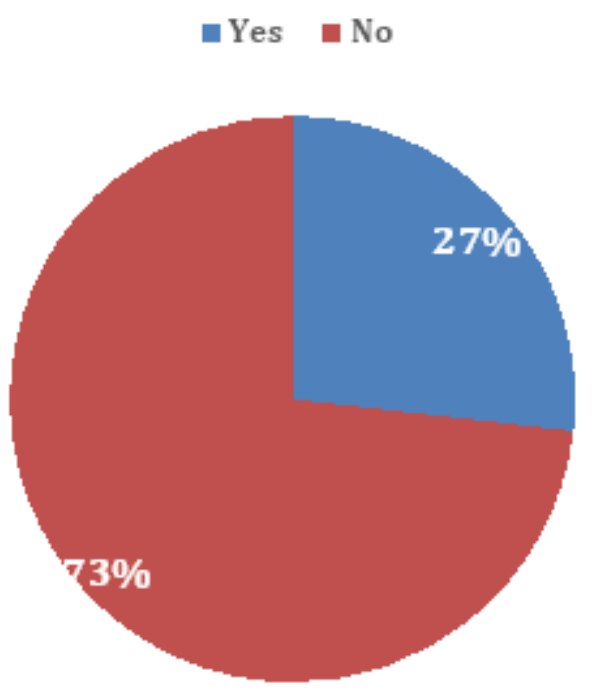

\section{Figure 7}

Figure 3.7: Figure showing the percentage of healthcare professionals who have children who participated in the study to assess the psychological effects of the COVID-19 pandemic on healthcare professionals, Khartoum state hospitals, February $2021(\mathrm{~N}=344)$

\section{Figure 8}

Figure 3.8: Levels of anxiety among healthcare professionals who participated in the study to assess the psychological effects of the COVID-19 pandemic on healthcare professionals, Khartoum state hospitals, February $2021(\mathrm{~N}=344)$

\section{Figure 9}

Figure 3.9: Levels of stress among healthcare professionals who participated in the study to assess the psychological effects of the COVID-19 pandemic on healthcare professionals, Khartoum state hospitals, February $2021(\mathrm{~N}=344)$ 


\section{WORK-FAMILY BALANCE LEVELS}

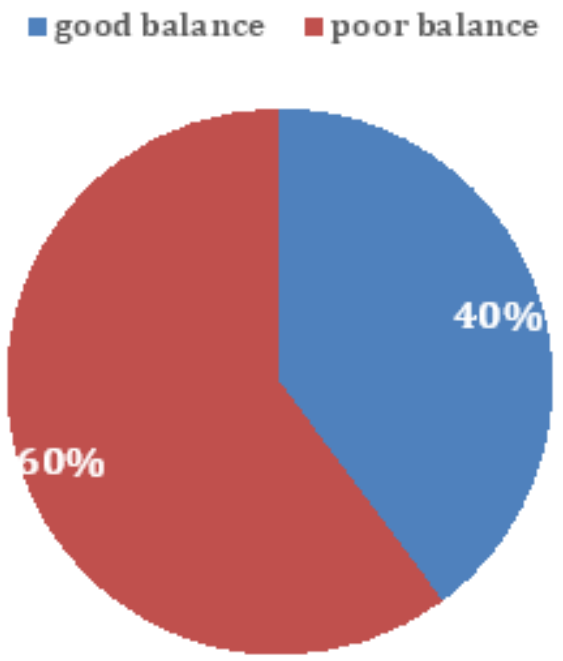

\section{Figure 10}

Figure 3.10: Work-family balance among healthcare professionals who participated in the study to assess the psychological effects of the COVID-19 pandemic on healthcare professionals, Khartoum state hospitals, February $2021(\mathrm{~N}=344)$

\section{COPING STRATEGIES}

Tuming To Religion (Praying more than often)

Problem Solving (Deal with problem)

Positive Attitude (Look for something good)

Avoidance/Denial Strategy

Seek Social Support (Get advice from someone)

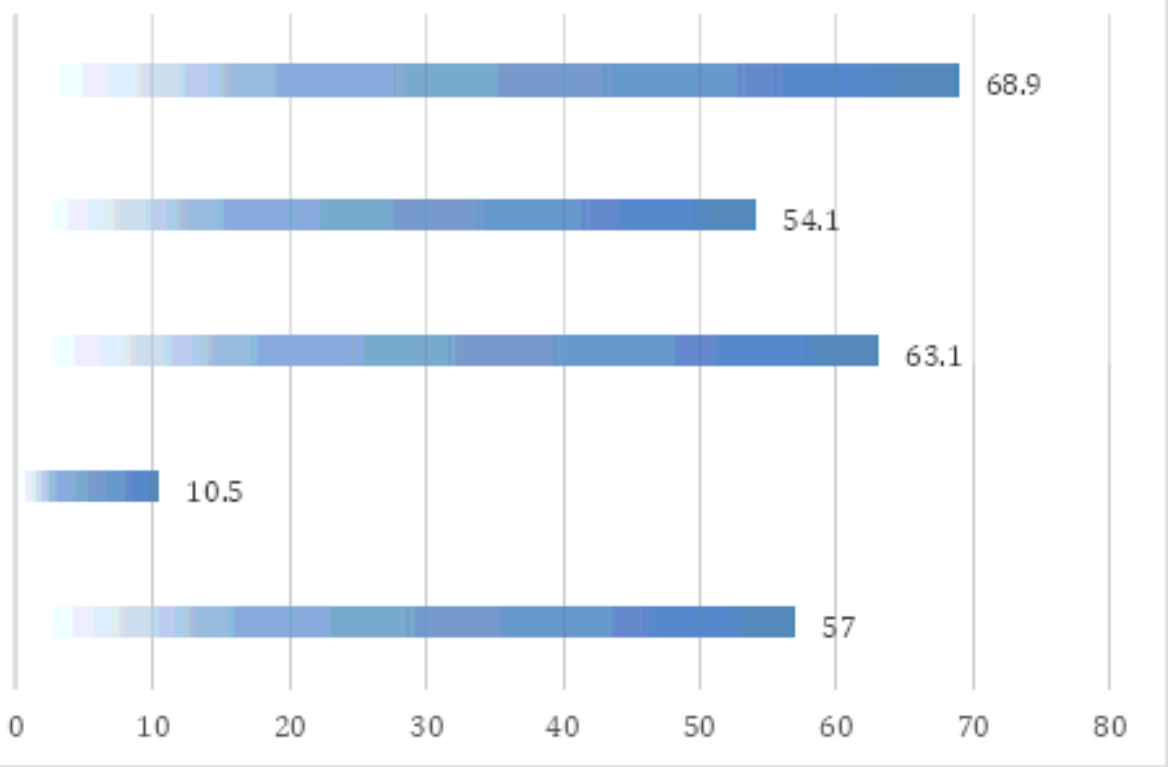

\section{Figure 11}

Figure 3.11: Figure showing the coping strategies used by healthcare professionals who participated in the study to assess the psychological effects of the COVID-19 pandemic on healthcare professionals, Khartoum state hospitals, February 2021 ( $N=344)$ 